\title{
Revisión sistemática de teorías de integración de sistemas de gestión normalizados
}

\section{Systematic review of integration theories of standardized management systems}

\section{Revisão sistemática de teorias de integração de sistemas de gestão normalizados}

Angélica Malagón Medina*

MARCALI S. A.

\section{RESUMEN}

Este artículo presenta los resultados de una revisión sistemática efectuada en Scopus, EBSCOhost y Unbis Thesaurus (United Nations Bibliographical Information System) y e-libro. La búsqueda consideró textos publicados entre el 2000 y el 2016. La recuperación de estos documentos se efectuó a través de palabras clave en inglés y español: integration of management systems, integration of management systems and systematic review, standards, industrial management. Se identificaron 998 documentos y se identificó que el 2014 es el año con mayor producción relacionada.
Dentro de la literatura científica, se pudo identificar que algunas revisiones sistemáticas de teorías de integración de sistemas de gestión son aplicadas a diferentes sectores económicos a nivel mundial, sin embargo, se tiene poca información del tema de investigación, los aportes son escasos; generando vacíos y dificultades para el análisis e interpretación de los elementos comunes de los aportes de teorías de integración de sistemas de gestión normalizados como ISO 9001, ISO 14001, OHSAS 18001.

Palabras clave: integración de sistemas de gestión, estándares, ISO.

Artículo de revisión. DOI: http://dx.doi.org/10.15332/s2145-1389.2018.0001.10

** Magíster en Calidad y Gestión Integral. Ingeniera industrial. Correo electrónico: angelikmalagon@hotmail.com 


\section{ABSTRACT}

This article presents the results of a systematic review carried out in Scopus, EBSCOhost and Unbis Thesaurus (United Nations Bibliographical Information System) and e-book. The search considered texts published between 2000 and 2016. The recovery of these documents was made through keywords in English and Spanish: Integration of management systems, Integration of management systems and systematic review, Standards, Industrial management. 998 documents were identified and it was identified that 2014 is the year with the highest related production. Within the scientific literature, it was possible to identify that some systematic reviews of theories of integration of management systems are applied to different economic sectors worldwide, however there is little information on the subject of research, contributions are scarce; generating gaps and difficulties for the analysis and interpretation of the common elements of the contributions of integration theories of standardized management systems such as ISO 9001, ISO 14001, OHSAS 18001.

Keywords: Integration of management systems, standards, ISO.

\section{RESUMO}

Este artigo apresenta os resultados de uma revisão sistemática realizada em Scopus, EBSCOhost e Unbis Thesaurus (Sistema de Informação Bibliográfica das Nações Unidas) e e-livro. A busca considerou textos publicados entre 2000 e 2016. A recuperação desses documentos foi realizada através de palavras-chave em inglês e espanhol: Integração de sistemas de gestão, Integração de sistemas de gestão e revisão sistemática, Normas, Gestão industrial. Foram identificados 998 documentos e foi identificado que o 2014 é 0 ano com a maior produção relacionada. Dentro da literatura científica, foi possível identificar que algumas revisões sistemáticas de teorias de integração de sistemas de gestão são aplicadas a diferentes setores econômicos em todo o mundo, porém há pouca informação sobre 0 tema de pesquisa, as contribuições são escassas; gerando vazios e dificuldades para a análise e interpretação dos elementos comuns das contribuições das teorias de integração de sistemas de gestão normalizados como ISO 9001, ISO 14001, OHSAS 18001.

Palavras-chave: integração de sistemas de gestão, padrões, ISO.

\section{INTRODUCCIÓN}

La integración de sistemas de gestión normalizados es un tema que ha tenido mayor incidencia e investigación a nivel mundial, como lo afirma Karapetrovic y Casadesús (2011), en un comienzo países como Estados Unidos y Japón asimilaban la adaptación de la norma de calidad y ambiente como un obstáculo no arancelario o prerrequisito para participar en subastas y licitaciones internacionales.

De acuerdo con Abad y Sánchez (2000) la implementación del sistema de salud y seguridad en el trabajo conllevo a grandes esfuerzos económicos y operativos dentro de las organizaciones, por lo mismo se crearon diferentes guías y documentos públicos que presentan bases para lograr dicha implementación como respuesta a la ausencia de un estándar a nivel internacional.

Según Guerrero (2012), en el 2006 la disponibilidad de la PASS 99 aportó beneficios como la reducción de costos de implementación, duplicidad de actividades dentro de las organizaciones, siendo un esquema de estandarización de sistemas integrados de gestión. Sin embargo, esta norma aún no es reconocida 
mundialmente, dejando una brecha para la consolidación de teorías que permita la integración de sistemas de gestión normalizados.

Esta investigación busca cubrir los vacíos en la literatura científica, siendo una fuente de consulta para toda el área académica: docentes, estudiantes e investigadores que desarrollen alguna investigación basada en la integración de sistemas; sirviendo como referencia para el marco teórico para su nuevo trabajo de investigación. Así mismo es tomado como herramienta de formación para nuevos estudiantes que se enfoquen en esta área de estudio.

\section{METODOLOGÍA}

La investigación se desarrolló bajo el enfoque epistemológico histórico hermenéutico, que hace referencia a la comprensión de las investigaciones publicadas en fuentes de información secundarias: libros, artículos, teorías, autores dentro del periodo histórico del año 2000 al año 2016. Además, se consideraron las recomendaciones dadas por Castellanos y Martínez (2000).

Con un tipo de investigación cualitativo, en donde se presenta la recolección de datos no estructurados, con una muestra teórica finita de acuerdo con los criterios y parámetros utilizados durante la revisión sistemática.

Para la revisión sistemática se tuvo en cuenta estudios similares que sirvieron de modelo, en donde se resaltan algunos autores como: Merce Bernardo, Alexandra Simonb, Juan José Taríc, José F. Molina Azorínc y Bernardo, M. A., Casadesús, M. A., Karapetrovic, S. B., Heras, I. C.

Los criterios y parámetros de selección de la revisión sistemática se encuentran limitados a la integración de sistemas de gestión de calidad NTC-ISO 9001:2015, ambiental NTC-ISO 14001:2015, salud y seguridad en el trabajo NTC-OHSAS 18001:2007, nombrados a continuación:

Selección de fuentes de información, se eligieron bases de datos científicas indexadas como Scopus, EBSCOhost y Unbis Thesaurus (United Nations Bibliographical Information System), e-libro.

Búsqueda inicial, se clasificaron los resultados de acuerdo con los tesauros, partiendo de una búsqueda basada en el título y el resumen de cada documento, en donde se confirmó la relación con el tema de investigación.

Selección de palabras de búsqueda, con el fin de tener mayor precisión y calidad en la búsqueda y recuperación de información, se interpretaron las palabras clave en un contexto técnico, mediante el uso de tesauros en español e inglés:

- Integration of management systems.

- Integration of management systems and systematic revierw.

- Standards.

- Industrial management.

Periodo histórico, se delimitó el periodo comprendido entre 2000 y 2016, tomando como referencia la base de datos Scopus, en donde, al analizar los resultados obtenidos en la búsqueda por palabras claves, la mayor cantidad de información se concentra en este periodo, siendo el año 2014 el tiempo de mayor publicación de este tipo de información con 998 documentos.

Las categorías de análisis facilitaron la compresión del tema de estudio a partir de la clasificación de terminología asociada dentro de categorías y subcategorías representadas en la figura 1, las cuales estuvieron relacionadas con el desarrollo de los temas a lo largo del documento. 
Figura 1. Mapa conceptual de categorías

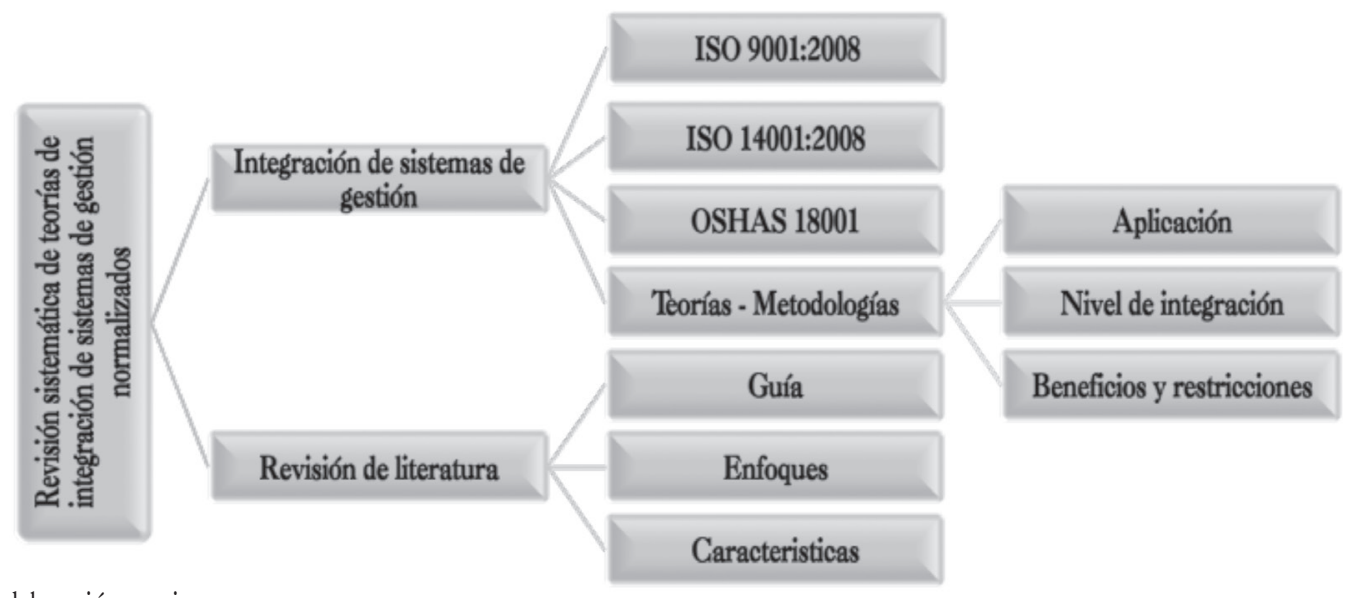

Fuente: elaboración propia.

Con el fin de examinar la calidad de la información encontrada en bases de datos, se recurrió al análisis bibliométrico.

Revisión journal ranking, mediante el uso de la Herramienta SCImago Journal Rank (SJR), con los siguientes filtros: revistas con mayor publicación dentro del área y países con documentos expuestos dentro de ellas. Para esta búsqueda, se limitó el tiempo de publicación al año 2016.

\section{RESULTADOS Y DISCUSIÓN}

Dentro de la búsqueda de información se presentaron dos periodos históricos, en donde se emplearon filtros de información con el fin de obtener las investigaciones relacionadas directamente con el tema de estudio.

Se realizó dos búsquedas en periodos históricos diferentes, con esta información se realizó un filtro de teorías por duplicados y que se encuentran fuera del objeto de la investigación, luego se seleccionó la información de acuerdo con las categorías de búsqueda, a continuación, se puede visualizar la cantidad de documentos recopilados en cada etapa.
Figura 2. Resumen búsqueda de documentos

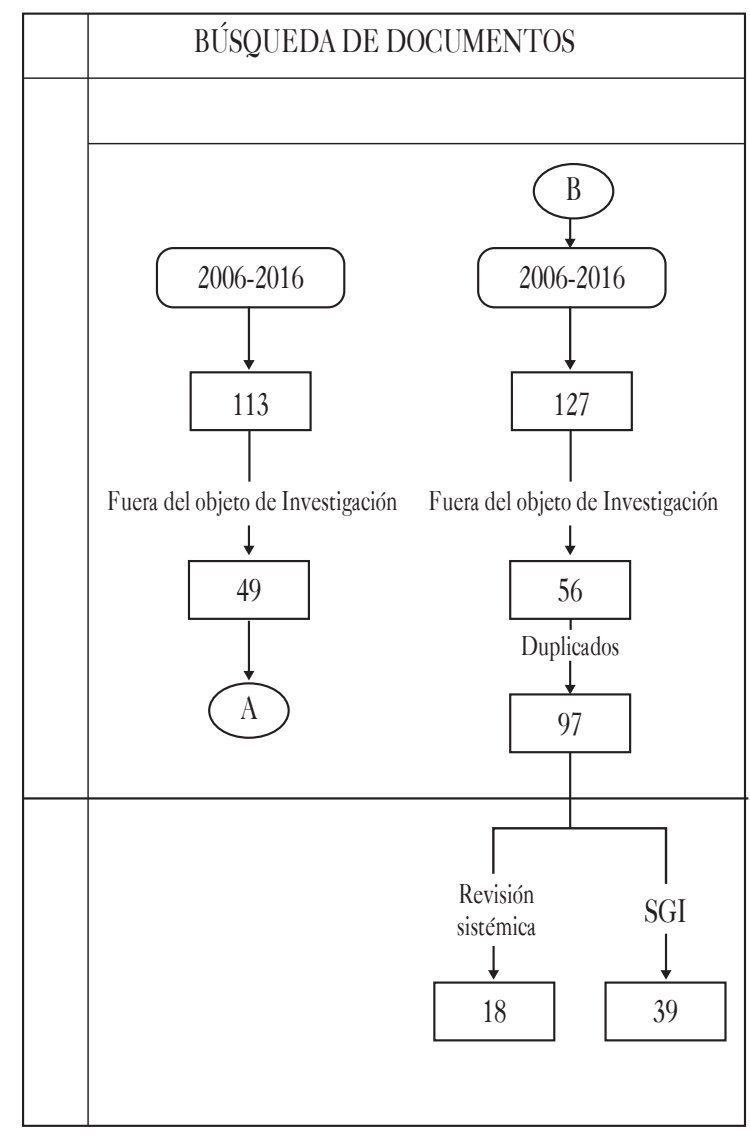

Fuente: elaboración propia. 
En total se analizaron 57 documentos los cuales se relacionan a continuación:

Tabla 1. Publicaciones analizadas

\begin{tabular}{|c|c|}
\hline \multicolumn{2}{|c|}{ Publicaciones analizadas } \\
\hline Karapetrovic y Willborn (1998a) & Bernardo, Casadesús, Karapetrovic y Heras (2012a) \\
\hline Karapetrovic y Willborn (1998b) & Bernardo, Casadesús, Karapetrovic y Heras (2012b) \\
\hline Hiller, Beer y Volm (2000) & Puente y Sánchez (2012) \\
\hline Ricart y Rodríguez (2000) & Sánchez, Palomino y Rivero (2012) \\
\hline Wilkinson y Dale (2000) & Simon, Karapetrovic, Casadesús (2012a) \\
\hline De Oliveira y Coelho (2002) & Simon, Karapetrovic, Casadesús (2012b) \\
\hline Ferguson, García y Bornay (2002) & Tarí, Molina y Heras (2012) \\
\hline Karapetrovic (2002) & Velásquez (2011) \\
\hline Karapetrovic y jonker (2003) & Velásquez (2012) \\
\hline Mackau (2003) & $\operatorname{AENOR~(2013)~}$ \\
\hline Stamou (2003) & Cordeiro y Morgado (2013) \\
\hline Zutshi y Sohal (2005) & De Oliveira y Coelho (2013) \\
\hline Camisón, Cruz y González (2006) & Simon, Bernanrdo, Karapetrovic, Casadesús (2013) \\
\hline Iyer (2006) & AENOR (2014) \\
\hline Heras, Bernardo y Casadesús (2007) & Durakbasa, Bas, Bauer y Kräuter (2014) \\
\hline Rocha, Searcy y Karapetrovic (2007) & Mohamad, Abdulllah, Mohammad y Kamaruddin (2014) \\
\hline Karapetrovic (2008) & Samani, Ismail, Leman y Zulkifli (2014) \\
\hline Bernardo, Casadesús, Karapetrovic y Heras (2009) & Bazil, Toktassynova, Mukhanov, Erenchinov, y Adilova (2015). \\
\hline López (2009) & Bernardo, Simon, Tarí y Molina-Azorín (2015) \\
\hline Peña, Rigol y Moreno (2009) & Cabrera, León, Puente, Rivera y Chaviano (2015) \\
\hline Tejada y Peña (2009) & Domingues, Sampaio y Arezes (2015) \\
\hline Farahani y Chitsaz (2010) & Gianni y Gotzamani (2015) \\
\hline Santana (2010) & Luo, Li y Li (2015) \\
\hline Adhikari (2010) & Owee, Hurairah, Sang y Rasli (2015) \\
\hline Bernardo, Casadesús, Karapetrovic y Heras (2011) & Bonilla y Martínez (2016) \\
\hline González (2011) & Jad'ud'ová, Zelený, Hroncová y Marková (2016) \\
\hline Karapetrovic y Casadesús (2011) & Luczak y Wolniak (2016) \\
\hline Soto (2011) & Rebelo, Santos y Silva (2016) \\
\hline Viloria (2011) & \\
\hline
\end{tabular}




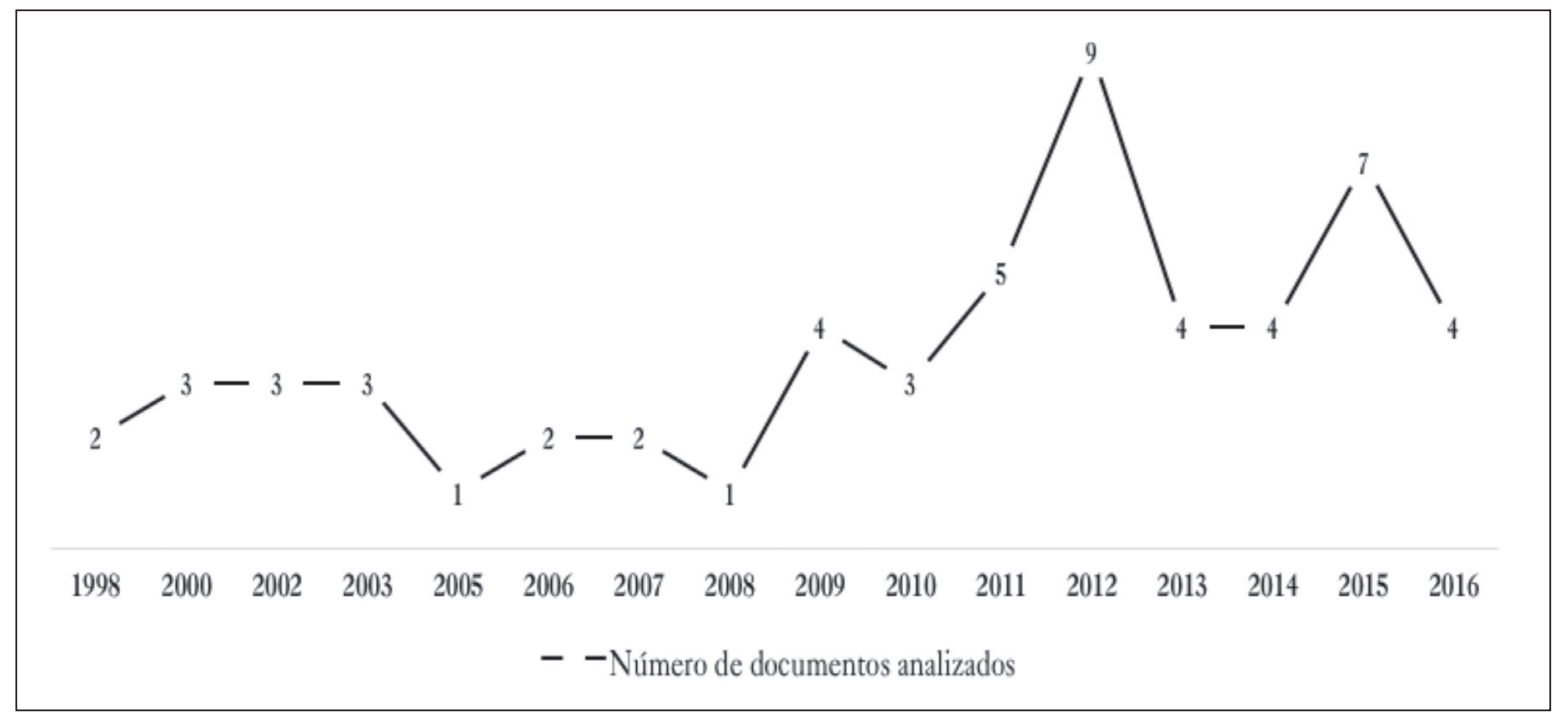

Fuente: elaboración propia.

\section{Criterios de recolección y análisis de la información de la revisión sistemática}

Para la distribución por idiomas, se obtuvo una participación del $74 \%$ de documentos obtenidos en el idioma inglés, seguido del idioma español con un total de 26 $\%$ documentos utilizados para el desarrollo del trabajo.

Este comportamiento se debe a que, en las bases de datos indexadas, disponen de la información más relevante filtrada por los tesauros en inglés, por lo tanto, se obtuvo poca información en el idioma "nativo".

La distribución por tipo de documento en donde se desarrolla el tema de estudio determinó las principales fuentes de búsqueda para futuras investigaciones. Se encuentran consolidadas en la siguiente tabla:

Tabla 2. Publicaciones por tipo de documento

\begin{tabular}{|l|c|c|}
\hline \multicolumn{1}{|c|}{ TIPO DOCUMENTO } & CANTIDAD & $\%$ \\
\hline Artículo & 21 & $54 \%$ \\
\hline Libros & 12 & $31 \%$ \\
\hline Tesis & 3 & $8 \%$ \\
\hline Revisiones & 2 & $3 \%$ \\
\hline Doc. Conferencia & 1 & $2 \%$ \\
\hline
\end{tabular}

Fuente: elaboración propia.
Es de aclarar que, al ser un tema relativamente nuevo, los argumentos de diferentes autores se exponen en artículos.

En la distribución por recursos bibliográficos consultados, se estableció que Scopus, e-libro y EBSCO son las bases de datos indexadas de las cuales proviene la mayor cantidad de información, y se relacionan directamente con el enfoque del tema.

La base de datos de la Universidad Santo Tomás (USTA), provee varias tesis en español relacionadas directamente con la integración de sistemas de gestión como:

- Cómo realizar una revisión sistemática y un metaanálisis.

- Integration of ISO 9001 and ISO 14001: A study of common elements.

\section{Elementos comunes de las teorías de SIG}

A continuación, se listan los aspectos básicos de relación identificados, en donde los autores enfatizan su necesidad como punto de partida para lograr una integración de sistemas de gestión normalizados. 
1. Estructura a través del ciclo de Deming (PHVA).

2. Implementación efectiva del sistema de gestión de calidad.

3. Compromiso de la dirección.

4. Alineación de los requisitos de las normas.

En la figura 3, se evidencia la cantidad de documentos con respecto al total de las investigaciones recolectadas, en donde se nombra cada uno de los aspectos básicos.

En esta figura se puede observar que el compromiso de la dirección y los requisitos de las normas tienen un aporte considerable, alcanzando más del $30 \%$ de participación; esta relación se puede asimilar con el desarrollo de la estructura de alto nivel, en donde se establecen los requisitos básicos de cada sistema de gestión bajo los lineamientos de la ISO.

Conceptos clave, dentro de recopilación de información, se observó que el 4 7\% de las investigaciones tienen un aporte del concepto de integración.
No obstante, el $53 \%$ de las investigaciones que no tienen un aporte del concepto de integración, mencionan aspectos favorables y dificultades de la integración de sistemas de gestión, por lo que se encuentra una equivalencia 0 variable que corrobora el enfoque del trabajo de investigación.

Figura 4. Concepto integración

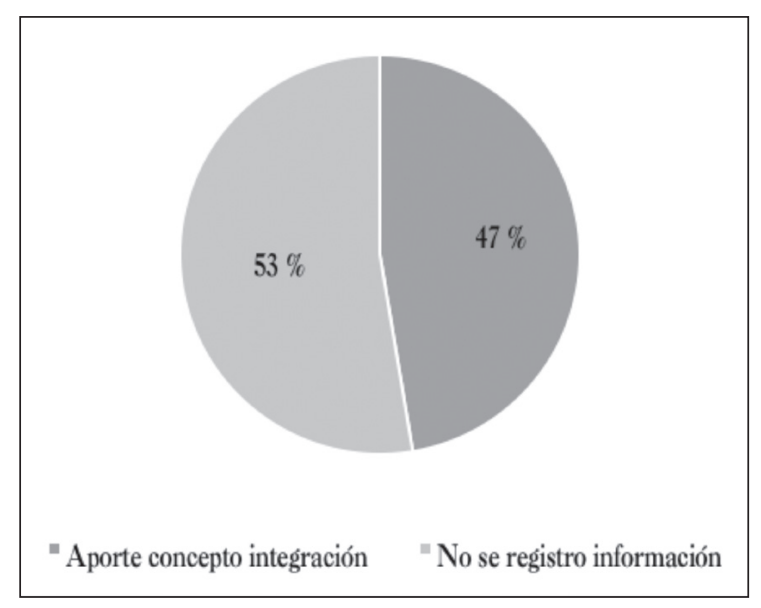

Fuente: elaboración propia.

Durante la recopilación de información de teorías de integración de sistemas de gestión normalizados en las investigaciones, se logró identificar algunas ventajas y dificultades de la integración de sistemas.

Figura 3. Aspectos básicos de relación

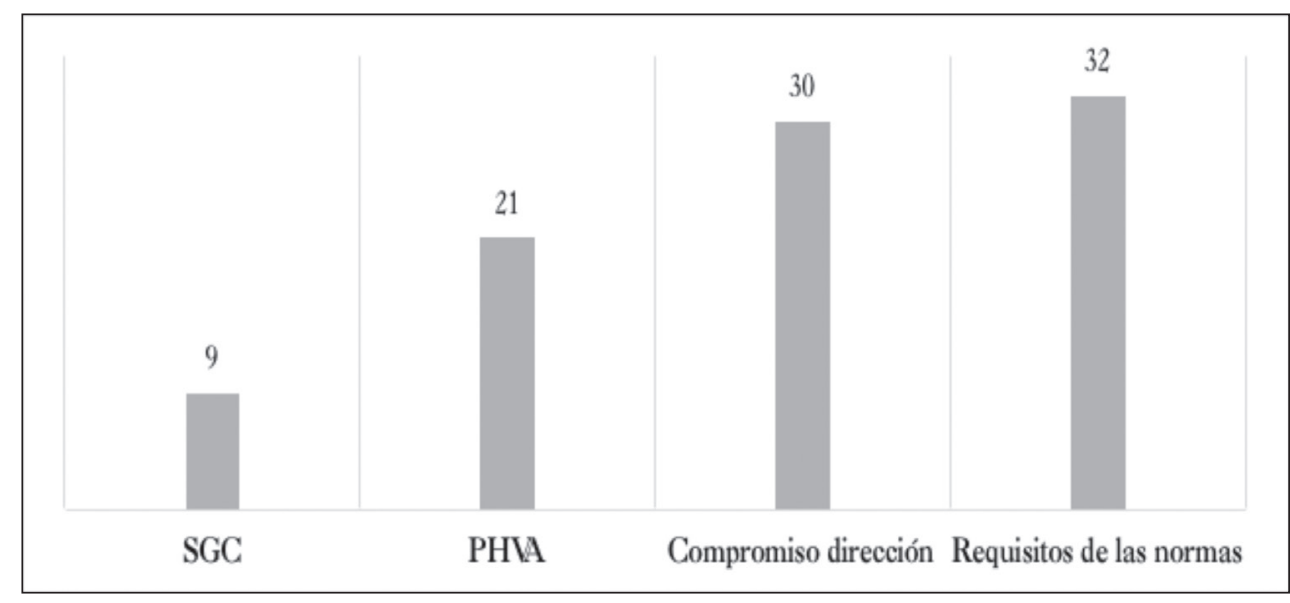

Fuente: elaboración propia. 


\section{Ventajas}

- Mayor motivación del personal.

- Mayor confianza de imagen positiva en el mercado.

- Mejor aprovechamiento de los recursos, impactos favorables en los resultados financieros.

- Mejora de la efectividad y eficiencia de la organización.

- Reducción de documentos, tiempos de auditoría y costos, unificación de elementos de las normas.

- Permite el uso y diseño de software.

\section{Dificultades}

- Falta de compromiso por la dirección, recursos insuficientes.

- Cultura empresarial desfavorable.

- Asignación del nivel de importancia a cada función técnica y a cada sistema.
- Formación adicional del personal encargado del SGI (Sistema de Gestión Integrado).

- Desalineación de los objetivos operacionales.

- Actualización de las normas a destiempo.

\section{Aportes de integración de sistemas de gestión normalizados}

Dentro de la información recolectada se analizaron los aportes de integración de sistemas de gestión, teniendo en cuenta: participación por autor, teorías expuestas, elementos básicos, equivalencias y descripción de cada teoría, detallado en los siguientes apartados.

Participación por autor, durante la revisión se observó que los autores con mayor participación en teorías son los autores que presentaban mayor ranking a nivel mundial en el análisis bibliográfico realizado.

Durante la consolidación de teorías de integración se identificaron 19 sin un nombre definido por el autor. Con el fin de establecer una forma fácil y ágil de identificación a través de un nombre temporal de las

Figura 5. Participación de autores

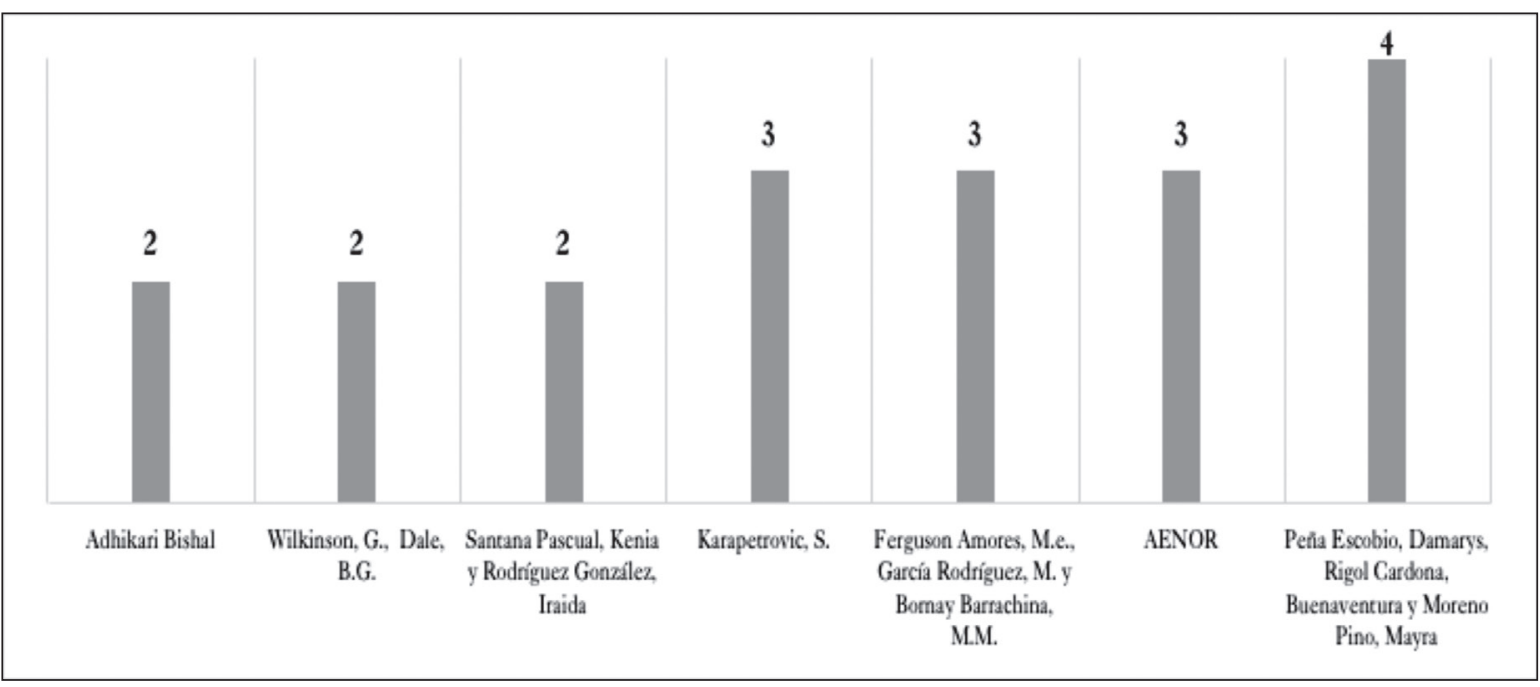

Fuente: elaboración propia. 
investigaciones recolectadas, se analizó la información por medio de equivalencias que buscan resaltar los elementos básicos y la descripción de estas.

Para esto se asignó una conexión o equivalencia que abarque el enfoque de cada aporte sin registro de nombre específico, nombradas a continuación:

- Integración por nivel organizacional y operativo.

- Relación con criterios de prevención de sistemas de gestión.

- Enfoque a indicadores claves en donde se evidencia la integración y sinergia.

- Momentos de implementación de los diferentes sistemas de gestión.

- Enfoque por procesos, manejo de estructura de alto nivel con fin de obtener resultados con mayor eficiencia.

- Integración por etapas de forma progresiva de acuerdo con la norma UNE 66172:2003.
- Relación con aspectos clave de la implementación de sistemas de gestión teniendo en cuenta la estructura de alto nivel.

Consolidación de teorías, al establecer la conexión y equivalencia de las teorías registradas sin nombre, se consolidó la información con las teorías que tenían el nombre definido por el autor, para tener una visión general de las teorías con mayor participación. A continuación, se representa la participación de cada teoría.

\section{CONCLUSIONES}

Criterios de selección y análisis de información. Para la revisión sistemática se tuvo en cuenta los siguientes criterios de selección y análisis de información, que cumplieron con el alcance y complejidad estimada, que permitieron la recolección de información que aportaron valor a la investigación.

- Bases de datos indexadas.

Figura 6. Nombre de teorías

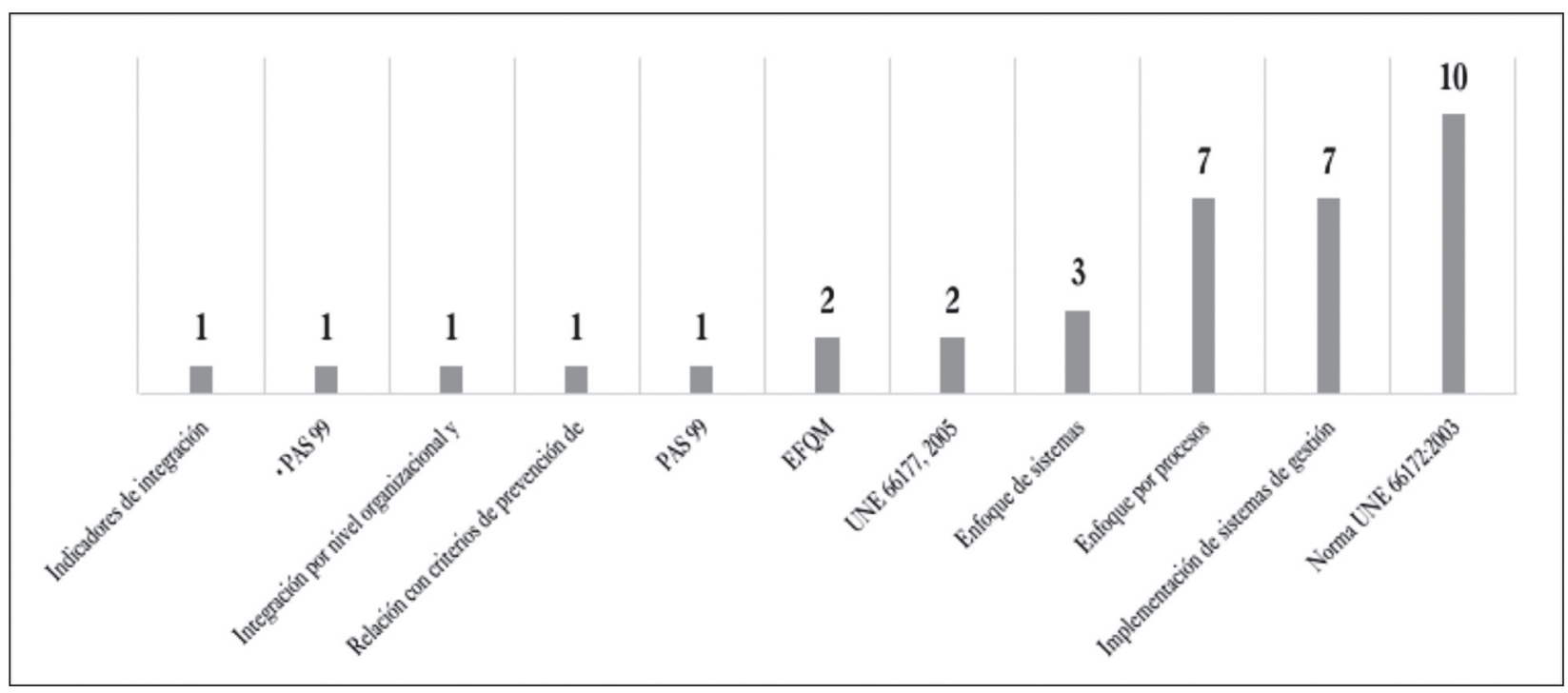

Fuente: elaboración propia. 
- Tesauros o palabras clave.

- Referenciación journal ranking.

- Periodo histórico.

- Categorías de búsquedas.

- Tipo de documento.

- Idioma.

Elementos comunes. Durante la revisión de las investigaciones recolectadas, se logró identificar elementos comunes como:

- La necesidad de tener el sistema de gestión de calidad implementado como base para integración de otros sistemas de gestión.

- La concepción del ciclo de Deming para la estructura de la integración de los sistemas de gestión.

- El compromiso de la dirección como principal componente la integración efectiva de sistema de gestión.

- Los requisitos comunes de las normas de sistemas de gestión.

En los elementos comunes de las teorías de integración se pudo identificar que el compromiso de la dirección y la alineación con los requisitos de las normas obtuvieron la mayor cantidad de citaciones dentro de las teorías de integración, esto es atribuido, en primera medida, a la estructura de alto nivel desarrollada por la ISO, la cual permite tener un estándar.

Un aspecto importante es que los requisitos de las normas compartidas pueden ser integrados en elementos básicos y manejados a través de módulos, es decir al agregar un nuevo sistema de gestión solo debe aumentar el número de módulos que aplican a una determinada situación particular o individual, por ejemplo el proceso y objetivo de auditoría en los sistemas de gestión son similares, solo se tienen que revisar algunos aspectos de recursos de cada sistema de gestión; lo que le da peso a la cantidad de citaciones de este aspecto dentro de las investigaciones.

Dentro de los aportes de teorías, los autores hacen énfasis en la efectividad de la integración de sistemas de gestión, teniendo la concepción en donde una organización solamente logrará alcanzar el éxito, si la dirección está comprometida con el desarrollo del sistema integrado de gestión, visto a través de la participación y capacitación de todo el personal que conforma la entidad.

Teorías de integración. En la consolidación de teorías de integración de sistemas de gestión, se recolectaron aportes de dicha teoría:

- Enfoque y principios TQM - EFQM.

- Enfoque de sistemas.

- Enfoque por procesos.

- Indicadores de integración.

- UNE 66177:2005.

- Norma UNE 66172:2003.

- Implementación de sistemas de gestión.

- Integración por nivel organizacional y operativo.

- Relación con criterios de prevención de sistemas de gestión.

De igual forma se identificaron los autores con mayor número de citaciones en las diferentes teorías de integración de sistemas de gestión, comprobando la información registrada dentro del análisis bibliométrico en el journal ranking. 
- Peña Escobio, Damarys, Rigol Cardona, Buenaventura y Moreno Pino, Mayra.

- Ferguson Amores, M. E., García Rodríguez, M. y Bornay Barrachina, M. M.

- Karapetrovic, $\mathrm{S}$.

- Santana Pascual, Kenia y Rodríguez González, Iraida.

- Wilkinson, G., Dale, B. G.

\section{REFERENCIAS}

Abad, P. J., y Sánchez, L. A. (2000). Aspectos clave de la integración de sistemas de gestión. España: AENOR - Asociación Española de Normalización y Certificación.

Adhikari, B. (2010). Integration of ISO 9001 and ISO 14001: A Study of Common Elements. American Psychological Association. University of Wisconsin stout. Recuperado de http://citeseerx.ist.psu.edu/ viewdoc/download?doi=10.1.1.390.947\&rep=rep1 \&type=pdf

AENOR. (2013). Guía rápida de correspondencia para la integración de sistemas de gestión. Madrid, España: AENOR - Asociación Española de Normalización y Certificación.

AENOR. (2014). Éxito de un sistema integrado. Madrid, España: AENOR - Asociación Española de Normalización y Certificación.

Bazil, G., Toktassynova, N., Mukhanov, B., Erenchinov, K., \& Adilova, S. (2015). Integration of QMS and BSC based on requirements. Paper presented at the 20155 th $^{\text {th }}$ International Workshop on Computer Science and Engineering: Information Processing and Control Engineering, WCSE 2015-IPCE.

Bernardo, M., Casadesus, M., Karapetrovic, S., \& Heras, I. (2009). How integrated are environmental, quality and other standardized management systems? an empirical study. Journal of Cleaner Production, 17(8), 742-750. doi:10.1016/j. jclepro.2008.11.003.

Bernardo, M., Casadesus, M., Karapetrovic, S., \& Heras, I. (2011). Relationships between the integration of audits and management systems: An empirical study. TQM Journal, 23(6), 659-672. doi:10.1108/17542731111175266.

Bernardo, M., Casadesús, M., Karapetrovic, S., \& Heras, I. (2012a). Integration of standardized management systems: Does the implementation order matter? International Journal of Operations and Production Management, 32(3), 291-307. doi:10.1108/01443571211212583.

Bernardo, M., Casadesús, M., Karapetrovic, S., \& Heras, I. (2012b). Do integration difficulties influence management system integration levels? Journal Of Cleaner Production, 21(1), 23-33. doi:10.1016/j. jclepro.2011.09.008.

Bernardo, M., Simon, A., Tarí, J. J., \& Molina-Azorín, J. F. (2015). Benefits of management systems integration: A literature review. Journal of Cleaner Production, 94, 260-267. doi:10.1016/j. jclepro.2015.01.075.

Bonilla, A. y Martínez J. (2016). Descifrando los niveles de integración de los sistemas integrados de gestión. SIGNOS - Investigación en sistemas de gestión, 8(2), 15 -37.

Cabrera, H. R., León, A. M., Puente, J. A., Rivera, D. N., y Chaviano, Q. N. (2015). La integración de 
Sistemas de Gestión Empresariales, conceptos, enfoques y tendencias. Ciencias de la Información, 46(3), 3-8.

Camisón, C., Cruz, S., y González, T. (2006). Gestión de la calidad: conceptos, enfoques, modelos y sistemas. Madrid: Pearson Prentice Hall

Casadesús, M., Heras, I., y Karapetrovic, S. (2009). Sistemas de gestión estandarizados: ¿existen sinergias? Revista europea de dirección y economía de la empresa, 18(2), 161-174.

Castellanos, Ó., y Martínez, C. (2000). Bases conceptuales y opción metodológica de la investigación en gestión. Cuadernos de Administración, 15(24), 105-132.

Cordeiro, T. B. C., \& Morgado, C. (2013). Audit methodology for environmental safety in offshore systems: An integrated approach of brazilian regulatory systems. doi: 10.4028/www.scientific.net/AMM.29 5-298.528.

De Oliveira, J. C., \& Coelho, D. A. (2002). The integration of the standards systems of quality management, environmental management and occupational health and safety management. International Journal of Production Research, 40(15), 3857-3866. doi:10.1080/00207540210155828.

De Oliveira, O. J. (2013). Guidelines for the integration of certifiable management systems in industrial companies. Journal of Cleaner Production, 57, 124-133. doi:10.1016/j.jclepro.2013.06.037.

Domingues, J., Sampaio, P., \& Arezes, P. (2015). Analysis of integrated management systems from various perspectives. Total Quality Management 8 Business Excellence, 26(11/12), 1311-1334. doi:10.1 080/14783363.2014.931064.

Durakbasa, N. M., Bas, G., Bauer, J. M., \& Kräuter, L. (2014). Development of an advanced metrology and intelligent quality management as a strategic approach in manufacturing industry. Paper presented at the 11th IMEKO TC14 Symposium on Laser Metrology for Precision Measurement and Inspection in Industry, LMPMI, (pp. 223-226).

Farahani, S. M., \& Chitsaz, G. (2010). Continual improvement with integrated management system. World Academy of Science, Engineering and Technology, 66, 1349-1353.

Ferguson, M, García, M y Bornay. (2002). Modelos de implantación de los sistemas integrados de gestión de calidad, el medio ambiente y la seguridad. Investigaciones Europeas de Dirección y Economía de la empresa, 8(1) 97 - 118.

Gianni, M., \& Gotzamani, K. (2015). Management systems integration: Lessons from an abandonment case. Journal of Cleaner Production, 86, 265276. doi:10.1016/j.jclepro.2014.08.023.

González, S. (2011). Sistemas integrados de gestión, un reto para las pequeñas y medianas empresas. Escenarios, 9(1), 69 - 89.

Guerrero, A. (2012). Implementación del sistema integrado de gestión en la empresa de diseño e ingeniería de Cienfuegos. (Tesis de maestría). Universidad Cienfuegos, Cienfuegos. Disponible en: http://www. eumed.net/libros-gratis/2013a/1330/index.htm.

Heras, I, Bernardo, M. y Casadesús, M. (2007). La integración de sistemas de gestión basados en estándares internacionales, resultados de un estudio empírico: realizado en la CAPV. Revista de dirección y administración de empresas, 14, 155-174.

Hiller, N., Beer, R., \& Volm, G. (2000). Introduction of a successful integrated management system. [Vorstellung eines erfolgreichen integrierten Managementsystems]. Melliand Textilberichte/International Textile Reports, 81(3), 211-212. 
Iyer, S. N. (2006). Integrated management system - implementing QHSE into projects from beginning to end. Paper presented at the 12th Abu Dhabi International Petroleum Exhibition and Conference, (ADIPEC). Meeting the Increasing Oil and Gas Demand through Innovation, 2, 864-869.

Jad'ud'ová, J., Zelený, J., Hroncová, J., \& Marková, I. (2016). Stakeholder management as part of integrated management system. Paper presented at the Production Management and Engineering Sciences-Scientific Publication of the International Conference on Engineering Science and Production Management. ESPM, 119-123.

Karapetrovic, S. (2002). Strategies for the integration of management systems and standards. TQM Maga\%ine, 14(1), 61-67. doi:10.1108/09544780210414254.

Karapetrovic, S. (2008). Augmentation and integration of standardised management systems. Paper presented at the Annals and Proceedings of the International DAAAM Symposium, (pp. 699-700).

Karapetrovic, S. y Casadesús M. (Septiembre, 2011). La integración de sistemas de gestión basados en estándares internacionales. [Integrating Management Systems: A dynamic study of Spanish firms]. En: XV Congreso de Ingeniería de Organización. [5th International Conference on Industrial Engineering and Industrial Management]. Cartagena, Colombia.

Karapetrovic, S., \& Jonker, J. (2003). Integration of standardized management systems: Searching for a recipe and ingredients. Total Quality Management and Business Excellence, 14(4), 451-459. doi:10.1080 /1478336032000047264.

Karapetrovic, S., \& Willborn, W. (1998a). Integrated audit of management systems. International
Journal of Quality and Reliability Management, 15(7), 694-711. doi:10.1108/02656719810218220.

Karapetrovic, S., \& Willborn, W. (1998b). Integration of quality and environmental management systems. TQM Magawine, 10(3), 204-213.

López, J. A. (2009). Los desafíos de la integración de sistemas de gestión. Celulosa y Papel, 25(1), 24-29.

Łuczak, J., \& Wolniak, R. (2016). Integration of quality, environment and safety management systems in a foundry. Metalurgïa, 55(4), 843-845.

Luo, H., Li, G., \& Li, C. (2015). Research on integration method of integrated management system. Open Automation and Control Systems Journal, 7(1), 1802-1807. doi:10.2174/18744444301507011802.

Mackau, D. (2003). SME integrated management system: A proposed experiences model. TQM Magasine, (1), 43-51. doi:10.1108/09544780310454448.

Mohamad, F., Abdulllah, N. H., Mohammad, M., \& Kamaruddin, N. K. (2014). Management systems integration for organisational sustainability: Quality, environmental, occupational health and safety, and energy.

Owee, T., Hurairah, A., Sang, C., \& Rasli, A. (2015). Development of quality management system and ISO 9001 integrated framework. Paper presented at the Engineering Technology, Engineering Education and Engineering Management-International Conference on Engineering Technology, Engineering Education and Engineering Management. ETEEEM, 1115-1119.

Peña, D., Rigol, B. y Moreno, M. (2009). Proyección de sistemas integrados de gestión. Recuperado de http:// www.eumed.net/ce/2009a/ecp.htm 
Puente, J., y Sánchez, A. (2012). Aspectos clave de la integración de sistemas de gestión. España: AENOR - Asociación Española de Normalización y Certificación.

Rebelo, M. F., Santos, G., \& Silva, R. (2016). Integration of management systems: Towards a sustained success and development of organizations. Journal of Cleaner Production, 127, 96-111. doi:10.1016/j. jclepro.2016.04.011.

Ricart, C. J. E., y Rodríguez, M. A. (2000). Coordinación de los sistemas de gestión de calidad, medio ambiente y salud laboral (parte I). Ediciones Deusto - Planeta de Agostini Profesional y Formación S. L.

Rocha, M., Searcy, C., \& Karapetrovic, S. (2007). Integrating sustainable development into existing management systems. Total Quality Management and Business Excellence, 18(1-2), 83-92. doi:10.1080/14783360601051594.

Samani, M. A., Ismail, N., Leman, Z., \& Zulkifli, N. (2014). Quality management system and risk management system: Similarities and possibilities for integration. doi:10.4028/www.scientific.net/ AMM.564.700.

Sánchez, C., Palomino, A., y Rivero, J. (2012). Manual para la integración de sistemas de gestion: calidad, medio ambiente y prevención de riesgos laborales. Madrid: FG Editorial. Fundación Confemetal.

Santana, P. K. (2010). Diseño e implantación del sistema integrado de gestión calidad, medio ambiente y seguridad y salud del trabajo. Cuba: D - Instituto Superior Politécnico José Antonio Echeverría. CUJAE.

Simon, A., Bernardo, M., Karapetrovic, S., \& Casadesus, M. (2013). Implementing integrated management systems in chemical firms. Total Quality $M a$ nagement and Business Excellence, 24(3-4), 294-309. doi:10.1080/14783363.2012.669560.
Simon, A., Karapetrovic, S., \& Casadesús, M. (2012a). Difficulties and benefits ofintegrated management systems. Industrial Management and Data Systems, 112(5), 828-846. doi:10.1108/02635571211232406.

Simon, A., Karapetrovic, S., \& Casadesus, M. (2012b). Evolution of integrated management systems in spanish firms. Journal of Cleaner Production, 23(1), 8-19. doi:10.1016/j.jclepro.2011.10.025.

Soto, O. (2011). Análisis económico sobre la generación de valor en las organizaciones colombianas certificadas, metodológicas y herramientas para la optimi ación de los sistemas de gestión. (Trabajo de maestría en Calidad y Sistemas de Gestión). Bogotá: Universidad Santo Tomas.

Stamou, T. (2003). Integrated management systems in small medium - sized enterprises: theory and practice. Thesis presented in part-fulfillment of the degree of Master of Science in accordance with the regulations of the University of East Anglia. School of Environmental Science. University of East Anglia. University Plain. Norwich.

Tarí, J. J., Molina, J. F., \& Heras, I. (2012). Benefits of the ISO 9001 and ISO 14001 standards: A literature review. Journal of Industrial Engineering and Management, 5(2), 297-322. doi:10.3926/jiem. 488.

Tejada, F., \& Peña, G. (2009). Reflexiones sobre las características constitutivas de la gestión integral. SIGNOS - Investigación en sistemas de gestión, 1(2), 79-93.

Velásquez, M. (2012). Planificación de un sistema de gestión de la calidad como plataforma para integrar otros modelos de gestión. SIGNOS - Investigación en sistemas de gestión, 4(2), 15-31.

Wilkinson, G., \& Dale, B. G. (2000). Management system standards: The key integration issues. 
Proceedings of the Institution of Mechanical Engineers, Part B. Journal of Engineering Manufacture, 214(9), 771-779.
Zutshi, A., \& Sohal, A. S. (2005). Integrated management system the experiences of three australian organisations. Journal of Manufacturing Technology Management, 16(2), 211-232. doi:10.1108/1741038 0510576840 . 